Europhysics Letters

PREPRINT

\title{
Breakdown of the Sonine expansion for the velocity distribution of Granular Gases
}

\author{
Nikolai V. Brilliantov ${ }^{1,2}$ and Thorsten Pöschel ${ }^{3}$ \\ 1 Institute of Physics, University of Potsdam, Am Neuen Palais 10, 14469 Potsdam, \\ Germany \\ 2 Department of Physics, Moscow State University, Vorobievy Gory 1, 119899 Moscow, \\ Russia \\ 3 Institut für Biochemie, Charité, Monbijoustraße 2, 10117 Berlin, Germany
}

PACS. 51.10.+y - Kinetic and transport theory of gases.

PACS. 45.70.-n - Granular systems.

PACS. 05.20.-y - Classical statistical mechanics.

\begin{abstract}
The velocity distribution of a granular gas is analyzed in terms of the Sonine polynomials expansion. We derive an analytical expression for the third Sonine coefficient $a_{3}$. In contrast to frequently used assumptions this coefficient is of the same order of magnitude as the second Sonine coefficient $a_{2}$. For small inelasticity the theoretical result is in good agreement with numerical simulations. The next-order Sonine coefficients $a_{4}, a_{5}$ and $a_{6}$ are determined numerically. While these coefficients are negligible for small dissipation, their magnitude grows rapidly with increasing inelasticity for $0<\varepsilon \lesssim 0.6$. We conclude that this behavior of the Sonine coefficients manifests the break down of the Sonine polynomial expansion caused by the increasing impact of the overpopulated high-energy tail of the distribution function.
\end{abstract}

Introduction. - The velocity distribution function of granular gases deviates from the Maxwell distribution, as first described by Goldshtein and Shapiro [1]. This deviation depends on the coefficient of restitution $\varepsilon$, which quantifies the loss of energy for a collision of two particles $i$ and $j$ :

$$
\vec{v}_{i}^{\prime}=\vec{v}_{i}-\frac{1+\varepsilon}{2}\left[\left(\vec{v}_{i}-\vec{v}_{j}\right) \cdot \vec{e}\right] \vec{e}, \quad \vec{v}_{j}^{\prime}=\vec{v}_{j}+\frac{1+\varepsilon}{2}\left[\left(\vec{v}_{i}-\vec{v}_{j}\right) \cdot \vec{e}\right] \vec{e} .
$$

Here $\vec{v}_{i}^{\prime}$ and $\vec{v}_{j}^{\prime}$ stand for the post-collisional velocities where the unit vector of the relative particle position at the collision instant is $\vec{e} \equiv\left(\vec{r}_{i}-\vec{r}_{j}\right) /\left|\vec{r}_{i}-\vec{r}_{j}\right|$.

The deviation from the Maxwell distribution may be described by a Sonine polynomials expansion [1-3]. This expansion is applicable to the main part of the velocity distribution, excluding the high-energy tails, which is known to be exponentially overpopulated [4].

So far it was silently accepted that the Sonine expansion is a converging series while the exponential tail does not noticeably contribute to the coefficients of this expansion. This assumption was supported by Direct Simulation Monte Carlo (DSMC) of the Boltzmann equation [5], as well as by Molecular Dynamics simulations of Granular Gases [6]. Up to now,

(C) EDP Sciences 
however, neither the region of validity of the Sonine expansion is known, nor the impact of the exponential tail on the convergence of this series.

Huthmann, Orza, and Brito [6] derived a system of equations for the Sonine coefficients from the Boltzmann equation and solved it perturbatively with the assumption that the Sonine coefficient $a_{k}$ is of the order $\mathcal{O}\left(\lambda^{k}\right)$ with $\lambda$ being a small parameter. For $\varepsilon \gtrsim 0.6$ they obtained rapid decrease of the Sonine coefficients with escalating order $k$, while for smaller values of $\varepsilon$ the high-order coefficients, $k \geq 3$, were not negligible and could be of the same order as the first non-trivial coefficient $a_{2}$. It was not evident, however, whether the Sonine polynomials expansion breaks down for $\varepsilon \lesssim 0.6$, or the perturbative approach based on the conjecture $a_{k} \sim \lambda^{k}$ was inadequate.

In the present study we derive the third Sonine coefficient, $a_{3}$, and address the convergence of the Sonine polynomials expansion analytically and numerically by means of DSMC. We show that the Sonine coefficients do not decrease with increasing $k \geq 3$ for $\varepsilon \lesssim 0.6$, i.e., the Sonine series diverges. We conclude that the breakdown of the Sonine expansion is caused by the increasing impact of the exponentially overpopulated tail for large dissipation.

Sonine polynomials expansion. - We consider a Granular Gas of particles of mass $m$ which interact with a constant coefficient of restitution $\varepsilon=$ const. and neglect their rotational degrees of freedom. We assume that the gas is in the homogeneous cooling state at number density $n=N / V$. Moreover, we assume that the velocity distribution function of the gas $f(\vec{v}, t)$ has acquired its scaling form $[1,4]$, i.e.

$$
f(\vec{v}, t)=\frac{n}{v_{T}^{d}(t)} \tilde{f}(\vec{c}), \quad \vec{c} \equiv \frac{\vec{v}}{v_{T}(t)},
$$

where $d$ is the system dimension and $v_{T}(t)$ is the thermal velocity due to the temperature $T(t)$,

$$
\frac{d}{2} n T=\int \frac{m v^{2}}{2} f(v) d \vec{v}=\frac{d}{2} n \frac{m v_{T}^{2}}{2} .
$$

For $\varepsilon=$ const. the Boltzmann equation reduces to two uncoupled equations, for the temperature and for the reduced distribution function $\tilde{f}(c)[1,4]$. The first equation reads

$$
\frac{d T}{d t}=-\frac{2}{d} g_{2}(\sigma) \sigma^{d-1} n v_{T} T \mu_{2},
$$

where $\sigma$ is the particle diameter. For $d=3$, the contact value of the pair distribution function is given by $g_{2}(\sigma)=(1-\eta / 2) /(1-\eta)^{3}$ with the packing fraction $\eta=n \pi \sigma^{3} / 6$, and

$$
\mu_{p} \equiv-\int d \vec{c}_{1} c_{1}^{p} \tilde{I}(\tilde{f}, \tilde{f})
$$

denotes the moments of the dimensionless collision integral $[2,3,7]$,

$$
\tilde{I}(\tilde{f}, \tilde{f}) \equiv \int d \vec{c}_{2} \int d \vec{e} \Theta\left(-\vec{c}_{12} \cdot \vec{e}\right)\left|\vec{c}_{12} \cdot \vec{e}\right|\left[\frac{1}{\varepsilon^{2}} \tilde{f}\left(\vec{c}_{1}^{\prime \prime}\right) \tilde{f}\left(\vec{c}_{2}^{\prime \prime}\right)-\tilde{f}\left(\vec{c}_{1}\right) \tilde{f}\left(\vec{c}_{2}\right)\right]
$$

The unit step function $\Theta(x)$ guarantees that only approaching particles collide, $\left|\vec{c}_{12} \cdot \vec{e}\right|$ gives the length of the collision cylinder and $\vec{c}_{1}^{\prime \prime}$ and $\vec{c}_{2}^{\prime \prime}$ denote the reduced velocities for the inverse collision, i.e., for the collision which results at the reduced velocities $\vec{c}_{1}$ and $\vec{c}_{2}$.

The second equation for the reduced distribution function reads

$$
\frac{\mu_{2}}{d}\left(d+c_{1} \frac{\partial}{\partial c_{1}}\right) \tilde{f}\left(\vec{c}_{1}\right)=\tilde{I}(\tilde{f}, \tilde{f}) .
$$


For the case of elastic collisions, $\varepsilon=1$, the resulting velocity distribution is the Maxwell distribution. Therefore, for sufficiently large coefficient of restitution, $\varepsilon \rightarrow 1$, we may assume that $\tilde{f}(c)$ is close to the Maxwellian, $\phi(c)=\pi^{d / 2} \exp \left(-c^{2}\right)$. This suggests to expand the (unknown) distribution function $\tilde{f}(c)$ around $\phi(c)$ in terms of orthogonal polynomials $S_{p}(x)$ :

$$
\tilde{f}(c)=\phi(c) \varphi(c)=\phi(c)\left[1+\sum_{p=1}^{\infty} a_{p} S_{p}\left(c^{2}\right)\right] .
$$

We chose $S_{p}(x)$ to be the Sonine polynomials (see, e.g., [8]):

$$
S_{p}(x)=\sum_{n=0}^{p} \frac{(-1)^{n}(p+1 / 2) !}{(n+1 / 2) !(p-n) ! n !} x^{n} .
$$

The first few of them, relevant for this study, read

$$
\begin{aligned}
& S_{1}(x)=-x+\frac{1}{2} d \\
& S_{2}(x)=\frac{1}{2} x^{2}-\frac{1}{2}(d+2) x+\frac{1}{8} d(d+2) \\
& S_{3}(x)=-\frac{1}{6} x^{3}+\frac{1}{4}(d+4) x^{2}-\frac{1}{8}(d+2)(d+4) x+\frac{1}{48} d(d+2)(d+4) .
\end{aligned}
$$

The expansion coefficients $a_{k}$ characterize the deviation of the distribution function from the Maxwell distribution, namely, they quantify the deviation of the moments of the distribution function, $\left\langle c^{p}\right\rangle \equiv \int d \vec{c} c^{p} \tilde{f}(c)$, from the corresponding values for the Maxwell distribution. The equations for the moments may be found multiplying both sides of Eq. (7) by $c_{1}^{p}$, integrating over $\vec{c}_{1}$, and using the orthogonality of the Sonine polynomials. This yields an infinite set of equations $[2,3,8]$,

$$
d \mu_{p}=\mu_{2} p\left\langle c^{p}\right\rangle, \quad p=2,4, \ldots
$$

Since $\left\langle c^{p}\right\rangle$ and $\mu_{p}$ are expressed in terms of the Sonine coefficients, this set of equations can be used to determine the Sonine coefficients and, thus, to find the velocity distribution. To close the set of equations, a cutoff of the series (8) is applied, that is, it is assumed that the Sonine coefficients $a_{k}$ with $k>k_{0}$ are negligible.

The first Sonine coefficient vanishes, $a_{1}=0$, according to the definition of temperature [1]. Since $\left\langle c^{2}\right\rangle=\frac{1}{2} d$, the first equation in Eq. (11) for $p=2$ gives the identity. The second equation in Eq. (11) for $p=4$ allows to find $a_{2}$ by expressing $\left\langle c^{4}\right\rangle, \mu_{2}$ and $\mu_{4}$ in terms of $a_{2}$ and neglecting all other Sonine coefficients $a_{3}, a_{4}, \ldots$ [1]. Therefore, the first non-trivial Sonine coefficient is $a_{2}$.

Second and third Sonine coefficients. - We write $\tilde{f}(c)=\phi(c)\left[1+a_{2} S_{2}\left(c^{2}\right)+a_{3} S_{3}\left(c^{2}\right)\right]$ for the velocity distribution function by assuming that $a_{4}, a_{5}, \ldots$ are negligible. Further, we write Eq. (11) for $p=4$ and $p=6$ and express all quantities in these two equations in terms of $a_{2}$ and $a_{3}$. In particular,

$$
\left\langle c^{4}\right\rangle=\frac{1}{4} d(d+2)\left(1+a_{2}\right), \quad\left\langle c^{6}\right\rangle=\frac{1}{8} d(d+2)(d+4)\left(1+3 a_{2}-a_{3}\right) .
$$

To find the moments $\mu_{2}, \mu_{4}$, and $\mu_{6}$ we recast Eq. (15) using the properties of the collision integral (see, e.g., [8]):

$$
\mu_{p}=-\frac{1}{2} \int d \vec{c}_{1} \int d \vec{c}_{2} \int d \vec{e} \Theta\left(-\vec{c}_{12} \cdot \vec{e}\right)\left|\vec{c}_{12} \cdot \vec{e}\right| \tilde{f}\left(c_{1}\right) \tilde{f}\left(c_{2}\right) \Delta\left(c_{1}^{p}+c_{2}^{p}\right),
$$


where $\Delta \psi\left(\vec{c}_{i}\right)=\psi\left(\vec{c}_{i}^{\prime}\right)-\psi\left(\vec{c}_{i}\right)$ denotes the variation of some quantity $\psi(\vec{c})$ in a direct collision. The evaluation of integrals of the form of Eq. (13) has been described in detail in [8]. Using this approach, analytical expressions for $\mu_{2}, \mu_{4}$, and $\mu_{6}$ may be obtained, which are rather cumbersome. Since it is expected that $a_{2} \gg a_{2}^{2}, a_{3} \gg a_{3}^{2}, a_{2} \gg a_{2} a_{3}$, and $a_{3} \gg a_{2} a_{3}$, we keep in these moments only linear terms with respect to $a_{2}$ and $a_{3}$ :

$$
\begin{aligned}
& \mu_{2}=\frac{\pi^{d / 2}}{\sqrt{2 \pi} \Gamma(d / 2)}\left(1-\varepsilon^{2}\right)\left[1+\frac{3}{16} a_{2}+\frac{1}{64} a_{3}\right] \\
& \mu_{4}=\frac{\pi^{d / 2}}{\sqrt{2 \pi} \Gamma(d / 2)}\left[T_{1}+T_{2} a_{2}+T_{3} a_{3}\right] \\
& \mu_{6}=\frac{\pi^{d / 2}}{\sqrt{2 \pi} \Gamma(d / 2)}\left[D_{1}+D_{2} a_{2}+D_{3} a_{3}\right]
\end{aligned}
$$

where

$$
\begin{aligned}
T_{1} & =\left(1-\varepsilon^{2}\right)\left(d+\frac{3}{2}+\varepsilon^{2}\right) \\
T_{2} & =\frac{3}{32}\left(1-\varepsilon^{2}\right)\left(10 d+10 \varepsilon^{2}+39\right)+(1+\varepsilon)(d-1) \\
T_{3} & =\frac{\left(1-\varepsilon^{2}\right)}{128}\left(10 \varepsilon^{2}+97\right)-\frac{(1+\varepsilon)(d-1)}{64}(21-5 \varepsilon) \\
D_{1} & =\frac{3}{4}\left(1-\varepsilon^{2}\right)\left[\left(d+\varepsilon^{2}\right)\left(5+2 \varepsilon^{2}\right)+d^{2}+\frac{19}{4}\right] \\
D_{2} & =\frac{3}{256}\left(1-\varepsilon^{2}\right)\left[1289-4\left(d+\varepsilon^{2}\right)\left(311+70 \varepsilon^{2}\right)+172 d^{2}\right]+\frac{3}{4} B(\varepsilon) \\
D_{3} & =-\frac{3}{1024}\left(1-\varepsilon^{2}\right)\left[2537+4\left(d+\varepsilon^{2}\right)\left(583+70 \varepsilon^{2}\right)+236 d^{2}\right]-\frac{9}{16} B(\varepsilon) \\
B & =(1+\varepsilon)\left[(d-3)\left(3+4 \varepsilon^{2}\right)+2\left(d^{2}-\varepsilon\right)\right] .
\end{aligned}
$$

For $a_{3}=0$, the above equations for $\mu_{2}$ and $\mu_{4}$ coincide with the equivalent equations in $[2,3]$.

Substituting Eqs. (12), (14) and (15) into Eqs. (11) for $p=4$ and $p=6$ we obtain the Sonine coefficients $a_{2}$ and $a_{3}$ in linear approximation. For $d=3$, the result reads

$$
\begin{aligned}
& a_{2}=-\frac{16}{b(\varepsilon)}\left(240 \varepsilon^{8}-480 \varepsilon^{7}+3312 \varepsilon^{6}-7424 \varepsilon^{5}+3510 \varepsilon^{4}-364 \varepsilon^{3}+895 \varepsilon^{2}+1934 \varepsilon-1623\right) \\
& a_{3}=-\frac{128}{b(\varepsilon)}\left(80 \varepsilon^{8}-160 \varepsilon^{7}+816 \varepsilon^{6}-1600 \varepsilon^{5}+154 \varepsilon^{4}+1548 \varepsilon^{3}-669 \varepsilon^{2}-386 \varepsilon+217\right) \\
& b(\varepsilon)=2800 \varepsilon^{8}-5600 \varepsilon^{7}+34800 \varepsilon^{6}-84480 \varepsilon^{5}-4410 \varepsilon^{4}+25716 \varepsilon^{3}+112155 \varepsilon^{2}-172458 \varepsilon+214357 .
\end{aligned}
$$

DSMC simulations. - To check the predictions of the theory and to study the behavior of higher Sonine coefficients we perform Monte Carlo simulations of the Boltzmann equation (DSMC) using $2 \times 10^{7}$ particles of unit mass. The coefficient of restitution was varied in the interval $\varepsilon \in(0.1,1)$ in steps of 0.01 . To obtain smooth data, for each value of $\varepsilon$ we performed 80 simulations and recorded the velocities of the particles when the system had reached a state with a scaling distribution function, Eq. (2). From these snapshots we computed the temperature and the moments of the reduced distribution functions evaluating the averages

$$
T=\frac{1}{3 N} \sum_{i=1}^{N} \vec{v}_{i} \cdot \vec{v}_{i}, \quad\left\langle c^{2 k}\right\rangle=\frac{1}{N} \sum_{i=1}^{N}\left(\frac{\vec{v}_{i} \cdot \vec{v}_{i}}{2 T}\right)^{k} .
$$




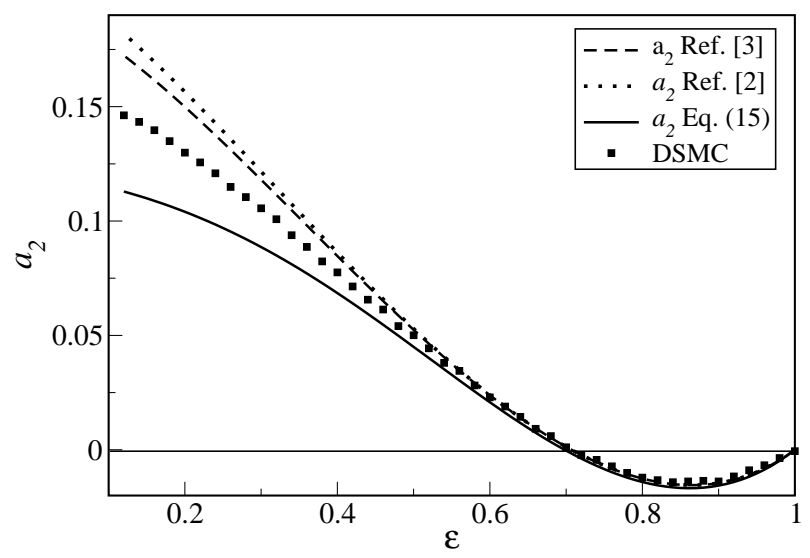

Fig. 1 - The second Sonine coefficient $a_{2}$ over the coefficient of restitution $\varepsilon$ as given by Eq. (16) where $a_{2}$ and $a_{3}$ are taken into account, $a_{2}(\varepsilon)$ from the linear theory, where only $a_{2}$ is taken into account [2], and $a_{2}(\varepsilon)$ from the corresponding non-linear theory [3], together with DSMC results.

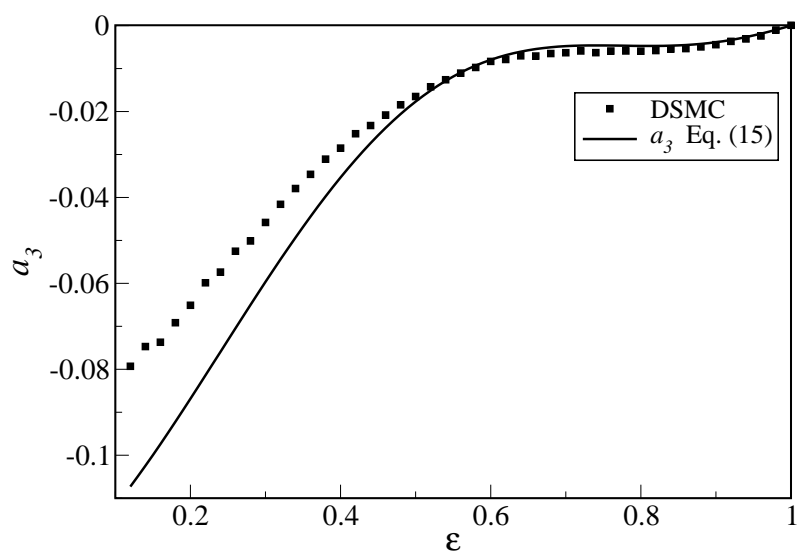

Fig. 2 - The coefficient $a_{3}$ over the coefficient of restitution $\varepsilon$ due to Eq. (16) and to DSMC.

The Sonine coefficients can be computed using the relation (see e.g. [8])

$$
\left\langle c^{2 k}\right\rangle=\frac{(2 k+1) ! !}{2^{k}}\left(1+\sum_{p=1}^{k}(-1)^{p} \frac{k !}{(k-p) ! p !} a_{p}\right) \text {. }
$$

Figure 1 shows $a_{2}$ as given by Eq. (16) (which takes $a_{3}$ into account), $a_{2}$ as it follows from the linear theory [2] and $a_{2}$ due to a non-linear theory [3] together with DSMC results. All approaches agree fairly well with the simulation results for small inelasticity, $\varepsilon \lesssim 0.6$, and deviate noticeably for larger dissipation. Figure 2 shows $a_{3}$ due to Eq. (16) together with the DSMC results. Again we see that the predictions of the new theory are in good agreement with the numerical results for $\varepsilon \gtrsim 0.6$. Similar numerical investigations of $a_{3}$ have been done by Brey et al. [5] for $\varepsilon>0.7$ and of $a_{3}, a_{4}, a_{5}$ by Nakanishi [9] for $\varepsilon>0.9$.

Higher Sonine coefficients. - In Fig. 3 we present the high-order Sonine coefficients as functions of the coefficient of restitution $a_{4}(\varepsilon), a_{5}(\varepsilon)$, and $a_{6}(\varepsilon)$. These coefficients are 


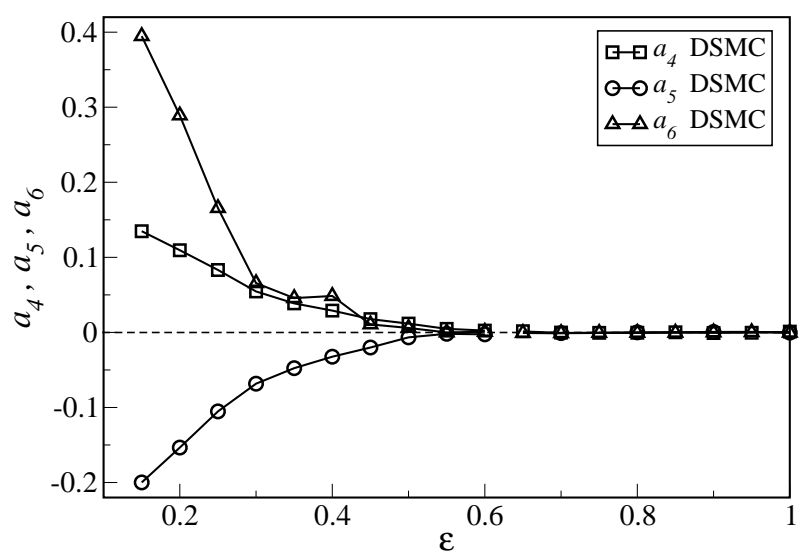

Fig. 3 - High-order Sonine coefficients as functions of the coefficient of restitution (symbols). The lines guide the eye.

very small for $\varepsilon \lesssim 0.6$, which indicates the convergence of the Sonine expansion. For larger inelasticity, however, the absolute values of $a_{p}, p=4,5,6$ increase with increasing $p$. Hence we conclude that for large inelasticity, $\varepsilon \lesssim 0.6$, the Sonine expansion does not converge. A similar result was reported in [6], where the Sonine coefficients were found from the Boltzmann equation under the assumption $a_{k} \sim \lambda^{k}(\lambda \ll 1)$.

What is the reason for the breakdown of the Sonine expansion with increasing inelasticity? This happens due to the increasing impact of the overpopulated tail of the velocity distribution function [4], which reads for $c \gg 1[2,4]$ :

$$
\tilde{f}(c) \sim B e^{-b c} ; \quad b=\frac{\pi^{(d-1) / 2} d}{\Gamma\left(\frac{d+1}{2}\right) \mu_{2}}
$$

where $\mu_{2}$ has been defined above, while the prefactor $B$ is unknown. For small $\varepsilon$ the exponential overpopulation starts for velocities that are not significantly larger than the thermal velocity. In this case the contribution to the moments $\left\langle c^{2 k}\right\rangle$ from the exponential tail rapidly grows with increasing $k$, which entails the corresponding growth of $a_{k}$ and ultimately, the breakdown of the Sonine expansion. Simulations show that at least in some range of $\varepsilon$ the main part of the distribution with $c \sim 1$ has a crossover to the tail distribution, Eq. (19), at $c \sim c^{*}[10]$. Assuming that the overpopulation of the tail starts at $c^{*} \sim b \sim 1 /\left(1-\varepsilon^{2}\right)$ [8] one can analyze the contribution from the exponential tail to the moments $\left\langle c^{2 k}\right\rangle$.

The breakdown of the Sonine expansion may be also understood from a simple mathematical argument: The Sonine expansion, Eq. (8), contains only even powers of the scaled velocity $c$. The tail of the distribution decaying as $\exp (-c), c \in\left(c^{*}, \infty\right)$, however, cannot be represented by a series in even powers of $c$. Therefore, for any value of $\varepsilon<1$, the presence of the asymptotic exponential tail must invalidate the Sonine expansion in the limit $c \rightarrow \infty$.

For $\varepsilon \lesssim 1$ the tail starts at rather large velocity $c^{*} \gg 1$, thus, only high-order terms of the Sonine expansion which are sensitive to large values of $c$ are affected and the corresponding high-order Sonine coefficients $a_{p}$ are large. Indeed, for $c \gg 1$, according to Eq. (8)

$$
\varphi(c)=\frac{\tilde{f}(c)}{\phi(c)}=\sum_{p} a_{p} S_{p}\left(c^{2}\right) \rightarrow B \exp \left(-b c+c^{2}\right) \simeq B \exp \left(c^{2}\right)=B \sum_{p} \frac{c^{2 p}}{p !}
$$


while the Sonine polynomials may be approximated by their leading-order terms:

$$
S_{p} \approx \frac{(-1)^{p} c^{2 p}}{p !}, \text { thus } a_{p} \rightarrow(-1)^{p} B \text { for } p \gg 1 .
$$

The distribution function itself, however, is extremely small for these velocities. For larger inelasticity, that is, smaller $\varepsilon$, the value of $c^{*}$ is not significantly larger than the thermal velocity. In this case, as shown in the present paper, already smaller Sonine coefficients such as $a_{3}$ to $a_{6}$ may diverge.

Nevertheless, the Sonine expansion remains a valuable tool for describing the main part of the velocity distribution, $\tilde{f}(c)$, for $c<c^{*}$ which is the range of interest in most cases. When considering the expansion up to $a_{6}$, the range $\varepsilon \in(0.6,1)$ seems to be a safe interval for applying the Sonine expansion. For higher-order expansions, however, the range of validity may be restricted to a smaller interval. A more quantitative discussion will be given in [10]

Conclusion. - We derived analytical expressions for the first two non-trivial Sonine coefficients as functions of the coefficient of restitution, $a_{2}(\varepsilon)$ and $a_{3}(\varepsilon)$, and show that the coefficient $a_{3}$ is not negligible as compared to $a_{2}$ as it was assumed in previous theories. We show that for small inelasticity, $0.6 \lesssim \varepsilon<1, a_{k}$ with $k \geq 4$ are significantly smaller than $a_{2}, a_{3}$ and may be neglected, that is, the Sonine expansion converges. For this interval of $\varepsilon$ the obtained theoretical values of $a_{2}$ and $a_{3}$ are in a good agreement with numerical results. We also find numerically the coefficients $a_{4}, a_{5}$, and $a_{6}$ for a wide range of the restitution coefficient, $0.1 \leq \varepsilon \leq 1$. For large inelasticity, $0<\varepsilon \lesssim 0.6$, the high-order Sonine coefficient are of the same order as $a_{2}, a_{3}$ and, hence, may not be neglected. The reason for this behavior is the significant contribution of the exponentially overpopulated tail to the moments of the velocity distribution function. This contribution rapidly growths with increasing inelasticity and the order of the moments, ultimately undermining the Sonine polynomial expansion.

\section{REFERENCES}

[1] A. Goldshtein and M. Shapiro. Mechanics of collisional motion of granular materials. Part 1: General hydrodynamic equations. J. Fluid Mech., 282:75, 1995.

[2] T. P. C. van Noije and M. H. Ernst. Velocity distributions in homogeneous granular fluids: the free and the heated case. Granular Matter, 1:57, 1998.

[3] N. V. Brilliantov and T. Pöschel. Deviation from Maxwell distribution in granular gases with constant restitution coefficient. Phys. Rev. E, 61:2809, 2000.

[4] S. E. Esipov and T. Pöschel. The granular phase diagram. J. Stat. Phys., 86:1385, 1997.

[5] J. J. Brey, D. Cubero, and M. J. Ruiz-Montero. Homogeneous cooling state of low-density granular flow. Phys. Rev. E, 54:3664, 1996.

[6] M. Huthmann, J.A. Orza, and R. Brito. Dynamics of deviations from the Gaussian state in a freely cooling homogeneous system of smooth inelastic particles. Granular Matter, 2:189, 2000.

[7] N. V. Brilliantov and T. Pöschel. Velocity distribution of granular gases of viscoelastic particles. Phys. Rev. E, 61:5573, 2000.

[8] N. V. Brilliantov and T. Pöschel. Kinetic Theory of Granular Gases. Oxford University Press, Oxford, 2004.

[9] H. Nakanishi. Velocity distribution of inelastic granular gas in a homogeneous cooling state. Phys. Rev. E, 67:010301R, 2003.

[10] T. Pöschel and N.V. Brilliantov. unpublished. 